The term "acute coronary syndrome" covers a spectrum of presentations, from unstable angina through to ST segment elevation myocardial infarction. There have been remarkable changes in the management of these conditions in the past two decades. With increasing emphasis on early reperfusion and prevention of left ventricular remodelling, echocardiography is assuming a prominent role in this area. It is non-invasive and relatively cheap, and is an ideal portable imaging technique. Newer imaging modalities, including myocardial contrast echo for the assessment of perfusion, hold great promise.

\title{
TECHNICAL ASPECTS
}

The first cardiac ultrasound machines displayed an ultrasound pulse versus depth on an oscilloscope screen. Incorporation of time as a dimension in the late 1960s converted this to a single line M mode display. M mode echocardiography is still in use, but has largely been supplanted by two dimensional (2D) echocardiography, ${ }^{1}$ which was developed in the 1970s. In the 1980s, spectral and colour Doppler were developed. The Doppler principle allows determination of the velocity and direction of blood flow, enabling assessment of valvar disease, shunts, and diastolic function. Transoesophageal echocardiography (TOE) was also introduced in the 1980s; initial probes were uniplane but multiplane probes are now routinely used, and there has been progressive transducer miniaturisation. TOE is very safe, with a mortality of less than $1 / 10000,{ }^{2}$ but not completely risk free (table 1) and should be performed by experienced physicians. It is relatively contraindicated in patients with oesophageal disease-for example, varices, stricture, oesophagitis, sclerodermaand may be hazardous in patients with severe coagulopathy or poor respiratory function; it is essential that the performing physician be aware of such conditions. Transthoracic echocardiography (TTE) and TOE are complementary techniques. While TOE avoids image degradation related to the chest wall and lungs, TTE may visualise anterior structures and the cardiac apex better. More windows for Doppler interrogation are available from TTE, and it is easier to make standard measurements. Both TTE and TOE are technically demanding procedures, and meticulous attention is required to ensure optimal images are obtained.

One of the most important recent technological achievements has been the development of harmonic imaging, which greatly improves endocardial definition. ${ }^{1}$ In conventional echocardiography, images are derived from ultrasound waves returned at the same frequency as the transmitted waves. However harmonic frequencies are also produced by the transmitted wave; in harmonic imaging, the machine uses the returning second harmonic to construct images. This improves image quality because there is less distortion of returned ultrasound waves (they are generated in the heart and only have to pass through tissue once), and few harmonics are produced close to the chest wall where many artefacts arise.

Digital echocardiography is an important advance ${ }^{3}$ of particular relevance to the coronary care unit. This is a process by which cardiac cycles are recorded in digital format rather than on videotape. It has huge practical advantages and is ideal for the temporal monitoring of left ventricular (LV) function as it enables side by side high quality images to be quickly available at multiple stations.

\section{COMPLICATIONS OF ACUTE MYOCARDIAL INFARCTION}

Echocardiography is the mainstay of diagnosis of mechanical complications of myocardial infarction (MI), ${ }^{4}$ and patients with unexplained haemodynamic deterioration should be immediately evaluated. It is important to recognise that TTE and TOE are complementary, and that TTE performed by an experienced echocardiographer may make an immediate diagnosis. In critically ill patients, image acquisition may be difficult; in these circumstances, TOE is extremely helpful.

Correspondence to: Dr Sally C Greaves,

Cardiology Department, Green Lane Hospital, Green Lane

West, Auckland, New

Zealand; sallyg@adhb.govt.nz

\section{Cardiac rupture}

Many LV ruptures cause sudden death. However rupture may be subacute, allowing time for intervention. Direct visualisation of the rupture is often difficult as it may be only a "slit" in the myocardium and the location of pericardial fluid may not correlate with the area of rupture. However, 
Table 1 Potential complications of transoesophageal echocardiography

- Respiratory depression

- Hypotension

- Arrhythmias

Laryngospasm/bronchospasm

- Dental trauma

- Pharyngeal/oesophageal/gastric trauma and perforation

- Aspiration

- Displacement of ET/nasogastric tubes

- Death

$E T$, endotracheal

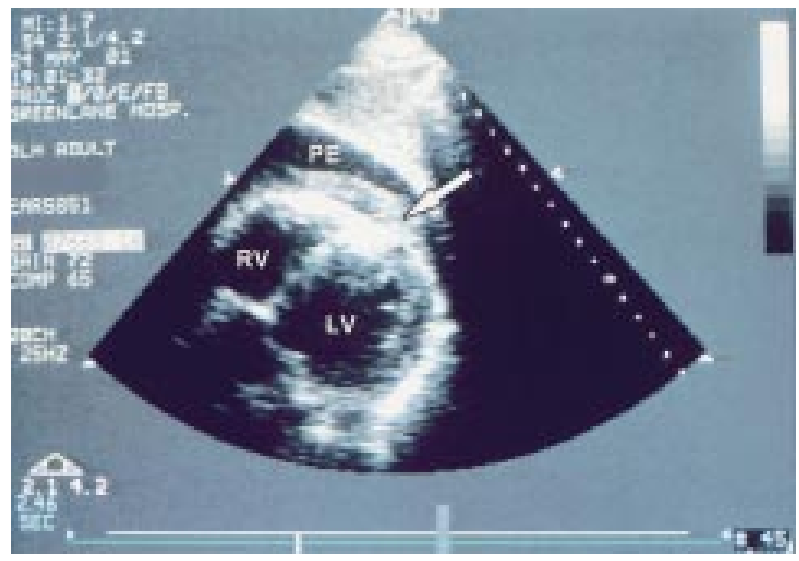

Figure 1 Subcostal long axis transthoracic image showing a pericardial effusion (PE) and intrapericardial thrombus (arrow) in a patient with left ventricular rupture post-infarction. LV, left ventricle; $\mathrm{RV}$, right ventricle.

intrapericardial thrombus is often present (fig l) and is very characteristic. It is rare to show flow into the pericardium. When caused by abrupt intrapericardial haemorrhage, the characteristic echocardiographic signs of tamponade may be absent, and diagnosis may rest on the volume of fluid and the clinical context. An LV pseudoaneurysm forms when the rupture is contained, so that a cavity outside the LV develops lined by pericardium and often thrombus. This can be distinguished from a true aneurysm by an abrupt interruption in the LV wall (as opposed to the smooth curve of a true aneurysm), a narrow neck, and low velocity bidirectional flow (fig 2A, B).

\section{Ventricular septal rupture}

Septal rupture may be difficult to distinguish clinically from mitral regurgitation (MR). From 2D echocardiography a discrete defect may be visible, but there may also be multiple serpiginous channels in the necrotic myocardium. The diagnosis can usually be made by TTE; experience is essential as the most useful views depend on the location of defect. Subcostal views are particularly useful in the critically ill, supine patient with inferior infarction (fig 3A). Small defects may not be visible but colour Doppler is very sensitive (fig 3B). Because these defects are often not discrete the degree of shunting may be difficult to evaluate. A large left-right shunt is characterised by hypercontractility of non-infarcted LV segments with a low LV stroke volume, high pulmonary artery flow velocities, and pulmonary hypertension. Posterior septal ruptures in particular tend to be complex and often associated with right ventricular infarction, which has an adverse prognosis.
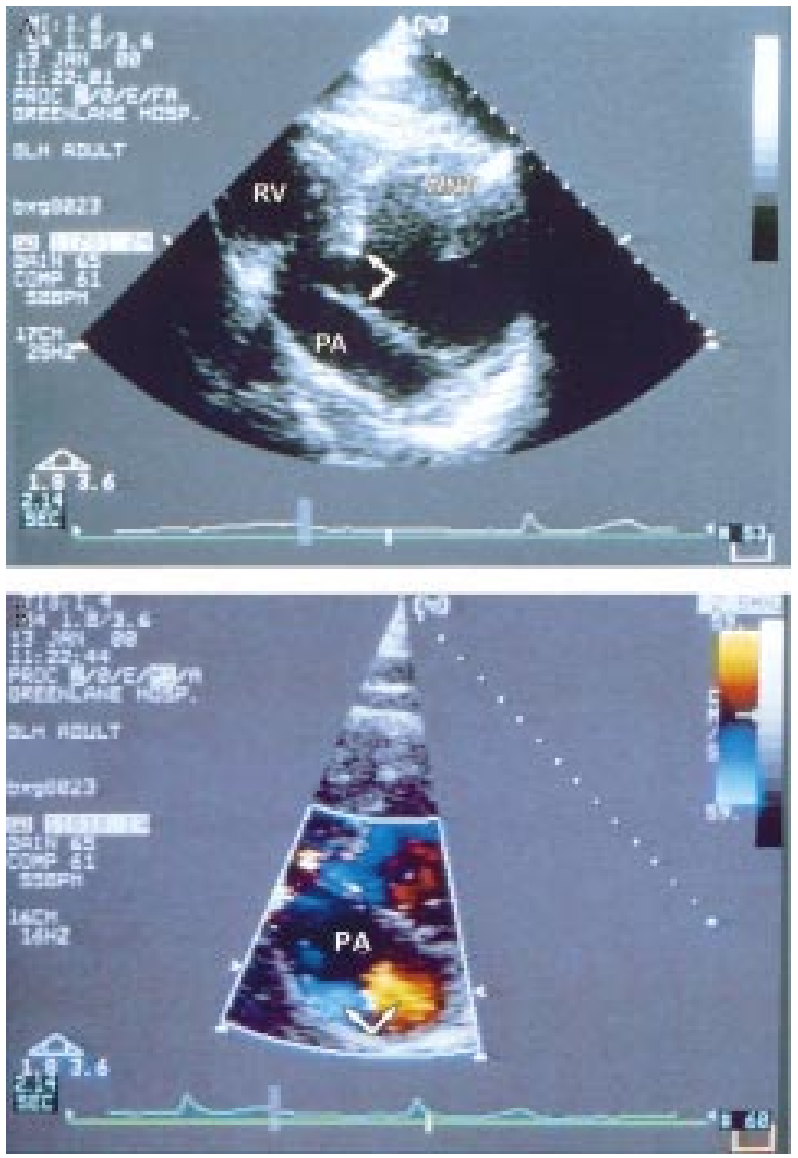

Figure 2 (A) Parasternal short axis transthoracic image showing a pseudoaneurysm of the inferior wall. The arrows show the characteristic abrupt disruption of the inferior wall and a narrow neck leading to the pseudoaneurysm. (B) Parasternal short axis transthoracic image (same patient as in A) showing bidirectional flow (arrows) from colour Doppler. Ant, anterior left ventricular wall, $\mathrm{RV}$, right ventricle. PA, pseudoaneurysm.

\section{Papillary muscle rupture}

Papillary muscle rupture is the most serious mechanism of MR in acute infarction. It usually involves the posteromedial muscle which is perfused from the posterior descending artery, whereas the anterolateral muscle has blood supply from both diagonal and circumflex arteries. Rupture of a papillary muscle head causes severe MR; rupture of the entire trunk is generally fatal. Transthoracic echocardiography is often suboptimal in evaluation-views of the papillary muscles are often limited, the MR jet is eccentric, and colour Doppler is influenced by the low LV/LA gradient in acute severe MR. TOE is a particularly suitable imaging modality ${ }^{4}$ and should be performed immediately if this diagnosis is suspected-it provides high resolution images of the papillary muscles (fig 4A) and accurate assessment of MR.

Rarely, multiple catastrophic mechanical complications of MI may occur in the same patient (figs $3 \mathrm{~A}$ and $4 \mathrm{~A}, \mathrm{~B}$ ).

\section{Aneurysm formation and left ventricular thrombus}

True aneurysms complicate transmural infarction and are caused by dilatation of an area of scar (fig 5). An aneurysm is defined as deformation of both the diastolic and systolic LV contours with dyskinesis in systole. TTE is a sensitive tool for the diagnosis but occasional false negatives occur, usually when the aneurysm involves a small part of the apex or the 

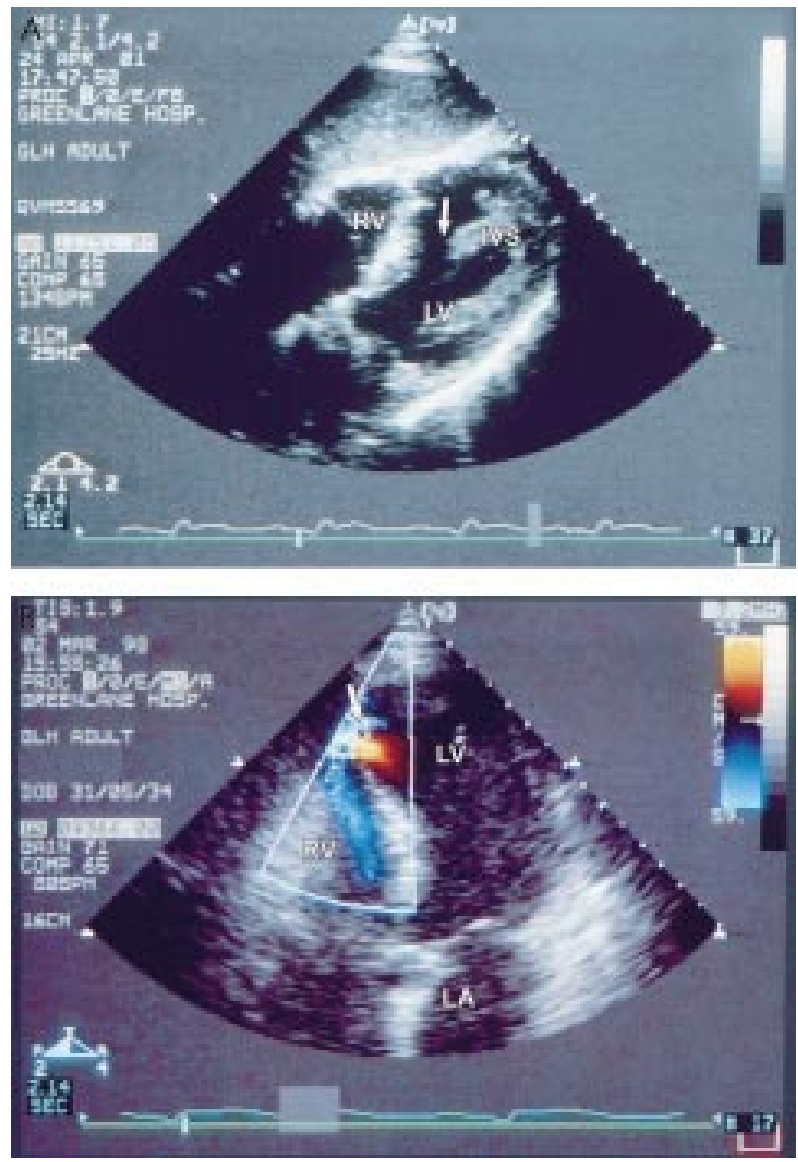

Figure 3 (A) Subcostal long axis transthoracic image showing a large post-infarction rupture (arrow) in the mid inferior ventricular septum (IVS). (B) Apical four chamber transthoracic image showing colour Doppler flow (arrow) through a postinfarction apical ventricular septal defect. LA, left atrium; LV, left ventricle; RV, right ventricle.

basal anterolateral wall. Aneurysm formation is a poor prognostic sign and is associated with congestive cardiac failure, arrhythmias, and thrombus formation. Left ventricular thrombi form in regions of stasis; they most commonly occur in the apex (fig 5) but may also be seen in lateral and inferior aneurysms. Certain echocardiographic characteristics (pedunculated and mobile thrombi) are associated with higher risk of embolisation. ${ }^{5}$ TOE may not visualise the apex as well as TTE.

\section{Pericarditis}

Echocardiography is a sensitive technique for the diagnosis of pericardial effusion; however the absence of fluid does not exclude pericarditis. In patients who are anticoagulated, intrapericardial thrombus may be recognised. Echocardiography can identify a site for percutaneous drainage if required and be used to monitor the procedure.

\section{Right ventricular infarction}

Recognition of right ventricular (RV) infarction is important, as it requires specific haemodynamic management. This syndrome occurs in more than $30 \%$ of patients with inferior MI but is rare in anterior infarction and in isolation. Many right coronary artery occlusions do not result in significant RV infarction due to the lower RV oxygen demand, higher oxygen extraction ration, greater systolic/diastolic flow ratio, and collateral supply. Right ventricular infarction may be diagnosed
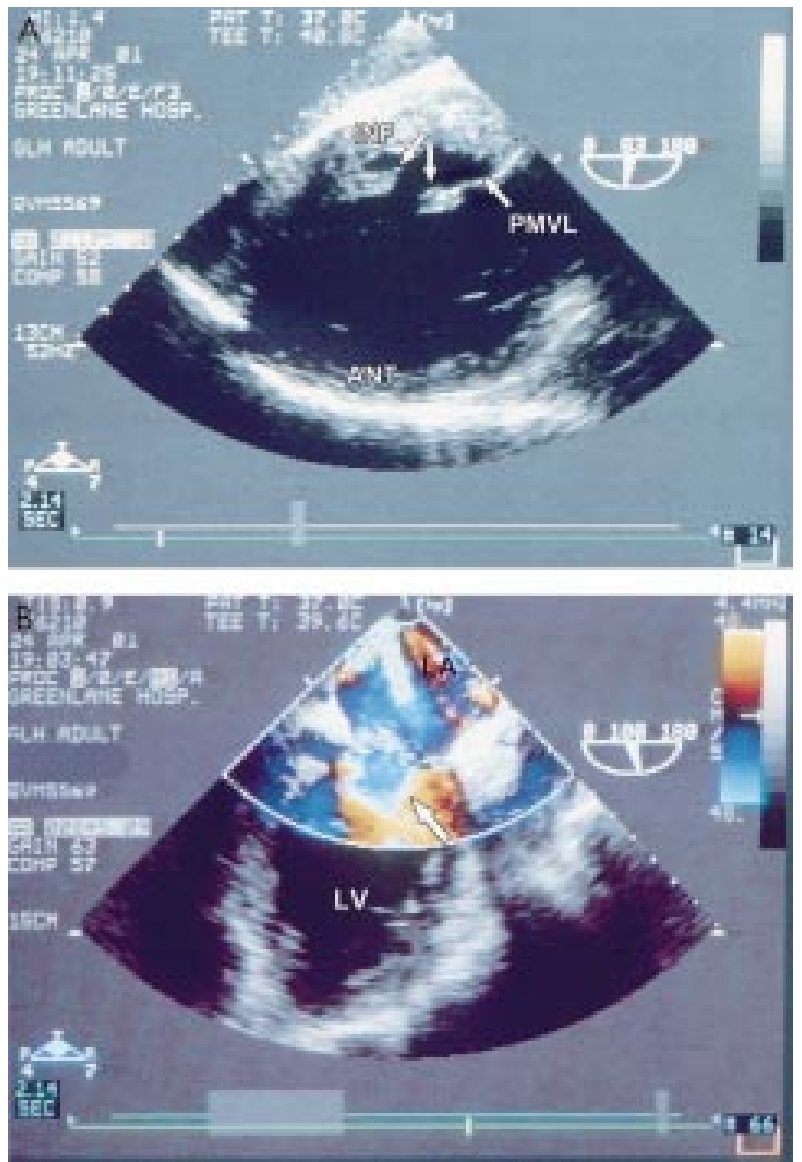

Figure 4 (A) Transgastric image showing rupture of the posteromedial papillary muscle postinfarction. The arrows point to the separated portions of the trunk of the papillary muscle.

(B) Transoesophageal image (same patient as in A) showing pronounced flow convergence from colour Doppler (arrow) as flow accelerates towards a regurgitant orifice; this is characteristic of severe mitral regurgitation. PMVL, posterior mitral valve leaflet; INF, inferior LV wall; ANT, anterior LV wall; LV, left ventricle; LA, left atrium.

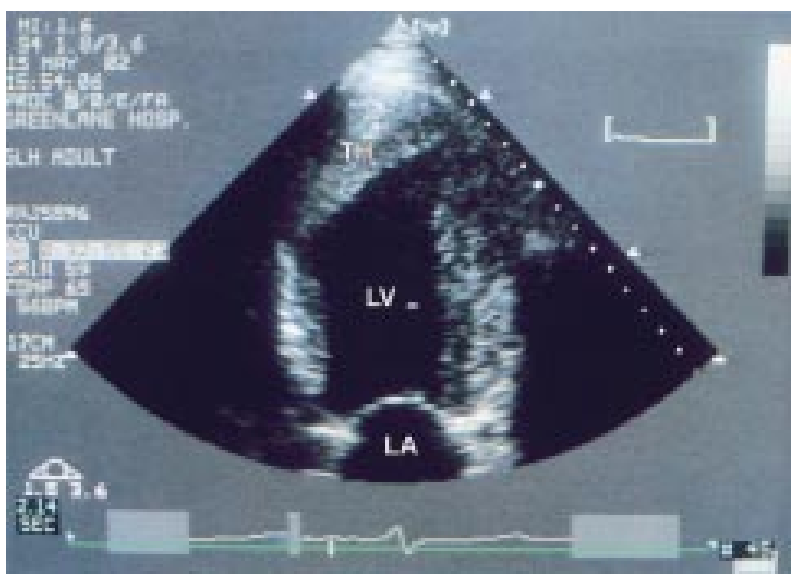

Figure 5 Apical four chamber transthoracic image showing extensive thrombus in an apical aneurysm. TH, thrombus; LV, left ventricle; $L A$, left atrium.

clinically and from right sided ECG leads, but echocardiography provides better assessment of the extent and severity. ${ }^{6}$ Right ventricular dysfunction may be global or regional; the 
RV outflow tract and apex are often spared, and hypercontractility in these regions may be a diagnostic clue. Echocardiography can evaluate complications of RV infarction. Functional tricuspid regurgitation is common; rarer complications are papillary muscle rupture and hypoxaemia from shunting through a patent foramen ovale caused by raised RA pressure.

\section{RISK STRATIFICATION IN THE CORONARY CARE} UNIT

Echocardiography is of tremendous value in risk stratification in MI and unstable angina. In general, it is helpful for these patients to have assessment of LV function before angiography. Echocardiography can provide a non-invasive biplane assessment, identify unsuspected valvar abnormalities, and evaluate right heart function. Left ventricular angiography may not be appropriate in critically ill patients and it is easier to obtain accurate information before invasive testing.

Patients with unstable angina and non-ST segment elevation MI are a heterogeneous group. Risk stratification is particularly relevant if conservative management is planned. Those patients with persistent wall motion abnormalities have more severe, chronic ischaemia and are at higher risk of adverse events. ${ }^{7}$ All patients with acute MI should ideally have early echocardiography. In some patients it may assist in diagnosis; it may also assist decision making if the appropriateness of reperfusion is uncertain, by demonstrating the localisation and extent of wall motion abnormality. Echocardiography is particularly important in those patients who are not obviously high risk; many patients without clinical evidence of LV dysfunction will have significant wall motion abnormalities. ${ }^{7}$ Patients with extensive regional abnormalities should have follow up echocardiography-this may detect early LV remodelling and other complications, and affect subsequent medical management.

Multiple indices of LV systolic and diastolic function have predictive value post-MI. Left ventricular wall motion score index (WMSI) is obtained by grading the motion of myocardial segments based on a standard model (table 2). It is particularly appealing, as while significant experience is required to interpret wall motion, it is highly reproducible. It reflects LV ejection fraction, but not LV size, so that quantitative measurements are also helpful. Left ventricular ejection fraction, ${ }^{7}$ end systolic volume, ${ }^{7}$ WMSI, ${ }^{8}$ and the presence of even mild $\mathrm{MR}^{9}$ are all early predictors of adverse outcome. Severe LV diastolic dysfunction tends to be associated with large infarctions, but a restrictive pattern is also independently associated with poor outcome. ${ }^{10}$ Although there are numerous factors which can alter filling patterns, Doppler echocardiography is very repeatable. The Doppler myocardial performance index is a more recently developed measurement combining indices of systolic and diastolic function. ${ }^{11}$ Repeated echocardiographic measurements have particular value, ${ }^{12}$ as they reflect LV remodelling, characterised by alterations in LV size, shape, and wall thickness. Remodelling may initially be compensatory in that it helps maintain LV stroke volume, but it progressively leads to increased LV wall stress, further dilatation, and eventually deleterious effects. Early remodelling and LV aneurysm formation is a particularly poor prognostic sign. Three dimensional echocardiography is more accurate than 2D echocardiography in the assessment of LV size and shape and may have a particular role in the evaluation of LV remodelling. It is a gold standard in research and is now coming into more routine clinical use, although the work involved in data acquisition and image reconstruction remains significant.

\begin{tabular}{|c|c|c|}
\hline Score* & Wall motion & Definition \\
\hline 0 & Hyperkinetic & $\begin{array}{l}\text { Increased endocardial inward movement } \\
\text { and systolic wall thickening }\end{array}$ \\
\hline 1 & Normal & $\begin{array}{l}\text { Normal endocardial inward movement and } \\
\text { systolic wall thickening }\end{array}$ \\
\hline 2 & Hypokinetic & $\begin{array}{l}\text { Reduced endocardial inward movement } \\
\text { and systolic wall thickening }\end{array}$ \\
\hline 3 & Akinetic & $\begin{array}{l}\text { Absence of endocardial inward movement; } \\
\text { no systolic wall thickening }\end{array}$ \\
\hline 4 & Dyskinetic & $\begin{array}{l}\text { Outward wall movement in systole with } \\
\text { absent wall thickening; often associated } \\
\text { with myocardial thinning and fibrosis }\end{array}$ \\
\hline
\end{tabular}

* The score for each segment is divided by the number of segments visualised to obtain the wall motion score index.

There are many newer techniques that can be utilised for evaluation of LV function and ischaemia, including tissue Doppler imaging, strain rate imaging, and tissue characterisation; the discussion of these is beyond the scope of this review.

Stress echocardiography has an important role post-MI. Although some centres use a stress imaging modality routinely post-MI, standard treadmill testing is adequate for many patients. Exercise echocardiography is appropriate for patients who can walk on a treadmill. Pharmacological stress echo is indicated primarily in patients unable to exercise adequately for non-cardiac reasons and for assessment of viability.

\section{ECHOCARDIOGRAPHY IN THE EMERGENCY ROOM AND CHEST PAIN UNIT}

The potential use of echocardiography for diagnosis of MI is based on observations of the effects of interruption of coronary flow. Left ventricular diastolic dysfunction occurs before systolic dysfunction; ECG abnormalities and chest pain are relatively late events. Segmental wall motion abnormalities seen by echocardiography correspond closely with coronary artery territories, although there is some variation depending on dominance of the right coronary artery and circumflex arteries and extent of "watershed" areas.

Optimal use of any diagnostic technique needs review of its cost effectiveness and impact on decision making, and use should always depend on the availability of resource and expertise. Sensitivity and specificity are inversely related; which is most important depends on the implications of missing the diagnosis versus making an incorrect diagnosis. Sensitivity and specificity are influenced by the pretest likelihood of disease (Bayes theorem). These are important considerations in examining the role of echocardiography in the emergency room as this is a much less selected patient group than in the coronary care unit. The problem is both economic and medico-legal. Many patients admitted with chest pain do not have an acute coronary syndrome whereas $5-10 \%$ of those who do are discharged. The challenge is to identify low risk patients without compromising the care of higher risk patients; the latter consideration is particularly relevant given the importance of early reperfusion. Chest pain units linked to the emergency room are an important approach to improving the quality and efficiency of care for these patients, with emphasis not only on reducing costs but on improving early diagnosis and triage to effective management.

There are many studies of echocardiography for MI diagnosis. ${ }^{13}{ }^{14}$ Some are in small, highly selected groups but most have found similar diagnostic sensitivity (approximately 
90-95\%). The negative predictive value is high (approximately $95 \%)$, but the positive predictive value is much lower and more variable. ${ }^{14}$ This may partly relate to interpretation difficulties in the presence of prior infarction, and aggressive interpretation of minor abnormalities by physicians anxious to avoid false negatives. From these studies, echocardiography appears more sensitive than standard criteria for the diagnosis of infarction; it is also sensitive for the diagnosis of myocardial ischaemia, but only if performed during pain. Echocardiography provides incremental prognostic information in the identification of patients at risk of cardiac events. However, if echocardiography is used alone, a small number of patients with subendocardial infarction will be discharged.

The study by Trippi and colleagues ${ }^{15}$ is an example of how echocardiography might be aggressively used in the emergency room. These authors enrolled 163 patients with no evidence of MI on initial cardiac markers or ECG, who were recommended for admission. If rest echocardiographic images were normal, dobutamine stress echocardiography was performed, initially supervised by a cardiologist and, in later stages, by a trained nurse. Echocardiographic images were transmitted by tele-echocardiography and interpreted off-site. In the first three stages, all patients were admitted. In the final stage, patients were discharged if the stress echocardiogram was negative. Average length of stay was only 5.4 hours. In the third and fourth stages recruitment was less selective, so that in the final phase mild residual chest pain, a non-diagnostic rather than normal ECG, and mild elevation of initial creatine kinase (CK) with normal CK-MB were permitted. The negative predictive value of dobutamine stress echocardiography was $98.5 \%$ based on final diagnosis, which was largely based on clinical follow up. There were two false negative results-one in a patient who was admitted in the third stage but discharged without a clinical diagnosis, and one in a patient who was discharged following a normal stress echocardiogram. The study is interesting because of the aggressive approach to achieving discharges, the use of tele-echocardiography and nursing supervision to avoid having a cardiologist on-site, and the choice of pharmacological stress to avoid the noise of a treadmill and the requirement for patient cooperation with exercise. However, most authors would argue that MI should be fully excluded by serial markers and ECGs, and that there be complete resolution of chest pain before stress testing.

In summary, echocardiography in the emergency room may facilitate early diagnosis and management in those patients with a high clinical suspicion of MI but a non-diagnostic ECG. It may also diagnose unstable angina if performed during pain. Aggressive use of rest and stress echocardiography can reduce admissions, but some false negatives will occur and

\section{Abbrevations}
CK: creatine kinase
2D: two dimensional
LV: left ventricular
MCE: myocardial contrast echocardiography
MI: myocardial infarction
MR: mitral regurgitation
RV: right ventricular
TIMI: thrombolysis in myocardial infarction
TOE: transoesophageal echocardiography
TTE: transthoracic echocardiography
WMSI: wall motion score index

Echocardiography in acute coronary syndromes: key points

- Transthoracic and transoesophageal echocardiography are complementary techniques with different strengths

- Transoesophageal echocardiography is very safe, but does have potential complications and should be performed by experienced physicians

- Hand held cardiac ultrasound devices are likely to be increasingly used in both the emergency room and coronary care unit but remain controversial

- Echocardiography for diagnosis of myocardial infarction is most helpful in patients with a high clinical suspicion but a normal or non-diagnostic ECG

- Patients with unexplained haemodynamic deterioration postinfarction should be referred immediately for echocardiography

- Echocardiographic indices of left ventricular systolic and diastolic function provide prognostic information in unstable angina and myocardial infarction

- Contrast echocardiography for left ventricular opacification may be helpful in the evaluation of cardiac function in "technically difficult" subjects

- Myocardial contrast echocardiography can evaluate the success of reperfusion and assess myocardial viability

- Intravenous myocardial contrast echocardiography is likely to become a routine clinical tool

small subendocardial infarctions may not be detected. These patients may be at a lower risk of complications but data on this are limited. False positives will also occur and differentiation of ischaemia from old infarction may be difficult; this is not of major importance, given that these patients are likely to be admitted. The chief logistical difficulty is the necessity for experienced staff and expensive equipment on a 24 hour basis.

Hand held, battery powered echocardiographic devices are now available. ${ }^{16}$ In many emergency rooms these devices are routinely used (a popular option as it avoids having to call the echocardiography laboratory); they are also used on ward rounds as "ultrasound stethoscopes" and for teaching. Studies show that they are more accurate than physical examination. However, they have limitations that can result in significant errors; Doppler functions on these devices are substantially inferior to the 2D imaging, although the quality will undoubtedly continue to improve. The optimal use of these devices and the implications for training and medicolegal issues remain controversial.

\section{CONTRAST ECHOCARDIOGRAPHY}

First generation contrast agents such as agitated saline have been available for many years and are still useful for detection of intracardiac shunts. However these agents do not opacify the LV, as the bubbles cannot survive passage through the lungs. Second generation contrast agents ${ }^{17}$ incorporate high molecular weight gases which are more stable and can traverse pulmonary capillaries. Microbubble properties depend on bubble size, shell composition, and the gas used.

When harmonic imaging is used, contrast agents achieve RV and LV opacification with improved image quality and reviewer confidence, ${ }^{17}$ and are of particular value in technically challenging subjects. They can also be used to enhance spectral Doppler signals; in patients with acute MI, they can occasionally be of value in other situations-for example, detection of myocardial rupture and LV thrombus. 


\section{Myocardial contrast echocardiography}

After many years of research, the assessment of coronary perfusion using myocardial contrast echocardiography (MCE) is starting to become a clinical reality. Visual assessment of myocardial perfusion from grey scale images is limited by the poor signal to noise ratio and the limited ability of the human eye to distinguish different shades of grey. Innovative ultrasound methods using harmonic imaging have been developed to exploit the interaction between microbubbles and ultrasound and enable assessment of perfusion. The use of contrast agents for LV opacification is very safe; the safety of MCE may depend on the type of contrast agent and mode of administration, and needs study in larger populations.

Myocardial perfusion has been extensively investigated by intracoronary injection, although no agents are specifically approved for intracoronary use. It is a reproducible and reliable technique for evaluation of the risk area after coronary occlusion, regional coronary flow reserve, myocardial viability, and the outcome of reperfusion. ${ }^{18}$

Intravenous MCE is an emerging technology, ${ }_{19}^{19}$ particularly in the assessment of reperfusion. The no-reflow phenomenon is a continuing challenge in cardiology. Angiographic TIMI (thrombolysis in myocardial infarction) flow grades provide prognostic information following reperfusion-TIMI 0-II flow is associated with poor recovery of contractile function, while TIMI III flow is associated with good recovery. However, up to $30 \%$ of segments with TIMI III flow have no reperfusion at microvascular level and in these regions there is myocardial necrosis. Multiple mechanisms, including microvascular disruption, endothelial and myocardial oedema, neutrophilic infiltration, and obstruction by thromboembolic debris may be involved. MCE is potentially ideal for assessment of reperfusion because the microbubbles stay largely in the capillaries, and $90 \%$ of the myocardial microvasculature is capillaries. Preserved microvascular integrity by MCE does not always translate into recovery, but absent perfusion has a high predictive value for lack of recovery and may identify patients requiring further management. Treatment options are limited at present but this is an area of active research.

There are limited data on MCE in acute MI, but it is being used in some centres. ${ }^{20}$ There are important technical questions that remain, but MCE could potentially become routine within the next five years. As with any new technique, clinical outcome and economic implications are important; indeed the proliferation of new technologies is a major factor in the rising costs of health care. Undoubtedly this technology will enter the coronary care and emergency room arenas. It could potentially be used to make the diagnosis of acute MI, define the area at risk, and then assess the results of reperfusion. There are exciting additional possibilities in the world of microbubbles; this is because they can be destroyed by ultrasound, and they can also serve as a vehicle for drug delivery to specific tissues, raising the possibility of ultrasound enhanced thrombolysis.

In summary, echocardiography is a critical modality in the diagnosis and management of patients with acute coronary syndromes. With the development of MCE it is anticipated that the routine use of echocardiography will extend into the cardiac catheterisation laboratory and operating room.

\section{REFERENCES}

1 Premawardhana U, Celermajer DS. Advances in echocardiography. Aust NZ J Med 2000;30:360-6.
- This is a basic, very readable review of some of the more recent advances in echocardiography imaging techniques and provides a good background for readers not familiar with the technology.

2 Daniel WG, Erbel R, Kasper W, et al. Safety of transesophageal echocardiography. A multicenter survey of 10,419 examinations. Circulation 1991;83:817-21.

3 Thomas JD. Digital storage and retrieval: the future in echocardiography. Heart 1997;78(suppl I):19-22.

4 Kishon Y, lqbal A, Oh JK, et al. Evolution of echocardiographic modalities in detection of post myocardial infarction ventricular septal defect and papillary muscle rupture: study of 62 patients. Am Heart J 1993;126/3 Pt 1):667-75.

- This study reports a large series of patients with septal and papillary muscle rupture postmyocardial infarction. It demonstrates the high sensitivity of transthoracic echocardiography for the detection of septal rupture and illustrates the use of transesophageal echocardiography.

5 Jugdutt BI, Sivaram CA. Prospective two-dimensional echocardiographic evaluation of $\mathrm{LV}$ thrombus and embolism after acute myocardial infarction. J Am Coll Cardiol 1989;13:554-64.

6 Goldberger JJ, Himelman RB, Wolfe CL, et al. Right ventricular infarction: recognition and assessment of its hemodynamic significance by two-dimensional echocardiography. J Am Soc Echocardiogr 1991;4:140-6.

7 Romano S, Dagianti A, Penco $M$, et al. Usefulness of echocardiography in the prognostic evaluation of non-Q-wave myocardial infarction. Am J Cardiol 2000;86(suppl 4A):43G-5G.

8 Peels KH, Visser CA, Dambrink JHE, et al on behalf of the CATS Investigators Group. Left ventricular wall motion score as an early predictor of left ventricular dilation and mortality after first anterior infarction treated with thrombolysis. The CATS investigators group. Am J Cardiol 1996; 77: $1149-54$

9 Feinberg MS, Schwammenthal E, Shlizerman L, et al. Prognostic significance of mild mitral regurgitation by color Doppler echocardiography in acute myocardial infarction. Am J Cardiol 2000;86:903-7.

10 Cerisano G, Bolognese L, Carrabba N, et al. Doppler-derived mitral deceleration time. An early strong predictor of LV remodeling after reperfused anterior acute myocardial infarction. Circulation 1999;99:230-6

- This is the first prospective study to correlate serial changes in LV volumes with changes in filling patterns and to show that a simple, early measurement of LV diastolic function can predict late left ventricular dilatation.

11 Moller JE, Sondergaard E, Poulsen SH, et al. The Doppler echocardiographic myocardial performance index predicts left-ventricular dilation and cardiac death after myocardial infarction. Cardiology 2001;95:105-11.

12 Korup E, Kober L, Torp-Pedersen C, et al on behalf of the TRACE Study Group. Prognostic usefulness of repeated echocardiographic evaluation after acute myocardial infarction. Am J Cardiol 1999;83:1559-62.

- This is a prospective study showing the value of serial echocardiographic measurements postinfarction. It is a relatively selected patient group but is of particular value in that patients with reinfarction were excluded. The wall motion index used in the study is derived in a different fashion from the more standard wall motion score index.

13 loannidis JPA, Salem D, Chew PW, et al. Accuracy of imaging technologies in the diagnosis of acute cardiac ischemia in the emergency department: a meta-analysis. Ann Emerg Med 2001;37:471-7.

- This is a meta-analysis of rest and stress echocardiography and nuclear studies from studies conducted between 1966 and 1998; although only three rest echocardiography studies are considered to meet the criteria specified, others are included in the sensitivity analyses.

14 Zabalgoitia $M$, Ismaeil $M$. Diagnostic and prognostic use of stress echocardiography in acute coronary syndromes including emergency department imaging. Echocardiography 2000;17:479-93.

- This is an excellent review of the use of both rest and stress echocardiography in the emergency room. It also has a good general review of the different modalities of stress echocardiographic testing and the relevant advances in imaging technology.

15 Trippi JA, Lee KS, Kopp G, et al. Dobutamine stress tele-echocardiography for evaluation of emergency department patients with chest pain. J Am Coll Cardiol 1997;30:627-32.

- This is a prospective, well designed study illustrating a possible, aggressive approach to using echocardiography to discharge patients early from the emergency room. It indicates methods by which some of the logistical difficulties might be overcome. It should be noted, however, that many authors would not be comfortable with the very early use of dobutamine stress echocardiography in some of these patients.

16 Seward JB, Douglas PS, Erbel R, et al. Hand-carried cardiac ultrasound $(\mathrm{HCU})$ device: recommendations regarding new technology. A report from the echocardiography task force on new technology of the nomenclature and standards committee of the American Society of Echocardiography. J Am Soc Echocardiogr 2002;15:369-73.

- This article contains recommendations from the American Society of Echocardiography regarding use of hand held cardiac ultrasound devices. It gives some general background, but concentrates primarily on clinical/legal issues. Physicians should become 
familiar with the issues involved; this paper also provides insight into considerations arising with the introduction of other forms of cardiovascular technology.

17 Mulvagh SL, DeMaria AN, Feinstein SB, et al. Contrast

echocardiography: current and future applications. J Am Soc Echocardiogr 2000;13:331-42.

- This is an American Society of Echocardiography position paper. It reviews contrast agents available and relevant imaging advances, concentrating on the clinical uses of these agents.

18 Czitrom D, Karila-Cohen D, Brochet $E$, et al. Acute assessment of microvascular perfusion patterns by myocardial contrast echocardiography during myocardial infarction: relation to timing and extent of functional recovery. Heart 1999;81:12-16.
19 Lepper W, Hoffmann R, Kamp O, et al. Assessment of myocardial reperfusion by intravenous myocardial contrast echocardiography and coronary flow reserve after primary percutaneous transluminal coronary angioplasty in patients with acute myocardial infarction. Circulation 2000;101:2368-74.

20 Kaul S. Myocardial contrast echocardiography in acute myocardial infarction: time to test for routine clinical use? Heart 1999;81:2-5.

- This is an editorial linked to a study of intracoronary myocardial contrast echocardiography. However, it is written by an international expert in this field, and provides an insightful view into the possible use of myocardial contrast echocardiography in the management of acute myocardial infarction. 